\title{
Estudio epidemiológico global del síndrome de Down
}

\author{
JULIO NAZER H. ${ }^{1}$, LUCÍA CIFUENTES O. ${ }^{2}$ \\ 1. Pediatra Neonatólogo Hospital Clínico Universidad de Chile. \\ 2. Instituto de Ciencias Biomédicas, Facultad de Medicina, Universidad de Chile.
}

\begin{abstract}
Global epidemiological study of Down's syndrome

Introduction: In 1967, the Latin American Collaborative Study of Congenital Malformations (LASCM) was created. Currently, 180 hospitals in 76 cities of 9 Latin American countries participate, accumulating data from over 4.5 M births. The Clinical Hospital of the University of Chile (HCUCH) entered the study in 1967, as did 12 other institutions later on. Objectives: Verify changes (increase) in frequency of Down's Syndrome (DS) in Chile and Latin America, and modification of frequency in those countries where elective abortion is permitted. Patients y Method: Three samples were evaluated: Births at HCUCH, active chilean hospitals and the 180 hospitals in 9 LA countries between 1972 and 2009. DS frequencies in Chile and other countries were compared through the International Clearinghouse for Birth Defects Monitoring System. Results: DS frequency at HCUCH increased significantly from 1.03 per thousand births in 1972 to $2.93 /{ }^{\circ 0}$ births 2009. Frequencies in all chilean hospitals are fairly homogeneous, all higher than average for LASCM, which is 1.88 per thousand. The chilean average is $2.47 /{ }^{\circ 0}$ por the period between $1998-2005$, with a range of 1.88 at HCUCH to 2.86 at the Hospital of Curicó. In the rest of the world, the rate of DS per birth has diminished significantly, being the lowest in Iran $\left(0.32{ }^{\circ 0}\right)$ and Spain (0.6/oo live births). Conclusion: Rate of DS births are higher in Chile than LASCM average $\left(2.47 /^{\circ 0}\right)$, with a trend to increase. This is true in Latin America, where the average for the period between 2001-2005 was 2.89 per thousand live births. In Europe and Asia, these rates have decreased to very low numbers, such as $0.32 /{ }^{\circ 0}$ in Iran and $0.6 /{ }^{\circ 0}$ in Spain.

(Key words: Down's syndrome, maternal age, congenital malformation, birth defect).

Rev Chil Pediatr 2011; 82 (2): 105-112
\end{abstract}

\section{RESUMEN}

Introducción: El ECLAMC (Estudio Colaborativo Latino Americano de Malformaciones Congenitas) fue creado en 1967. Actualmente, lo integran 180 hospitales de 76 ciudades de 9 países Latino Americanos y ha acumulado más de 4,5 millones de nacimientos. El Hospital Clínico de la Universidad de Chile (HCUCH) ingresó en 1967 y después otros 12 establecimientos chilenos. Objetivos: Verificar si la frecuencia de síndro-

Trabajo recibido el 18 de octubre de 2010, devuelto para corregir el 15 de noviembre de 2010, segunda versión el 14 de diciembre de 2010, aceptado para publicación el 24 de enero de 2011.

Correspondencia a:

Julio Nazer $\mathrm{H}$.

E-mail: jnazer@redclinicauchile.cl 
me de Down (SD) está aumentando en Chile y Latino América y como se ha visto modificada en los países en que está permitido el aborto electivo. Pacientes y Método: Se estudió 3 muestras: Todos los nacimientos del HCUCH desde 1972 a 2009; de los hospitales chilenos activos y de los 180 hospitales de 9 países Latinoamericanos. Se comparó las frecuencias de SD de Chile y del ECLAMC con otros países que participan en el Internacional Clearinghouse for Birth Defects Monitoring System. Resultados: La frecuencia del SD en el HCUCH aumentó significativamente de 1,03 por mil nacimientos en 1972 a 2,93 por mil en 2009. Las frecuencias en los hospitales chilenos son muy homogéneas, todas más altas que el promedio del ECLAMC: 1,88 por mil. El promedio chileno para el período 1998-2005 fue de 2,47 por mil. Con un rango de 1,88 por mil del HCUCH y 2,86 por mil del Hospital de Curicó. En el resto del mundo se ha asistido a una disminución significativa de las tasas de SD, siendo las más bajas la de Irán: 0,32 por mil y España 0,60 por mil nacimientos vivos. Conclusión: En Chile, las tasas de SD son mayores al promedio del ECLAMC 2,47 por mil existiendo una tendencia al aumento de ellas lo mismo que en Latino América, donde el promedio para el período 20012005 fue de 2,89 por mil. En Europa y Asia las tasas han disminuido a cifras extremas, Irán 0,32 por mil y España 0,60 por mil.

(Palabras clave: Síndrome de Down, Edad materna).

Rev Chil Pediatr 2011; 82 (2): 105-112

\section{Introducción}

El ECLAMC (Estudio Colaborativo Latino Americano de Malformaciones Congénitas) fue creado en 1967 como un Programa de investigación clínico-epidemiológico de factores de riesgo en la etiología de las malformaciones congénitas, utilizando una metodología casocontrol. A su vez es miembro del International Clearinghouse for Birth Defects Monitoring Systems. En el período 1967-2007 había registrado 300000 casos y sus respectivos controles sanos. Habiendo acumulado 4567809 nacimientos en 180 hospitales de 76 ciudades Latinoamericanas. Su función principal es la de monitorizar las frecuencias con que se presentan las malformaciones congénitas en general y de cada una en particular a lo largo del tiempo. El Hospital Clínico de la Universidad de Chile (HCUCH) inició un registro de malformaciones congénitas, como miembro del ECLAMC) en Septiembre de 1969. Desde esa fecha se ha mantenido en forma ininterrumpida y a cargo del mismo médico coordinador. Hemos comunicado nuestros hallazgos, en varias publicaciones ${ }^{1-6}$ Hemos observado que muchos defectos congénitos han aumentado su prevalencia al nacimiento, especialmente aquellos diagnósticos dependientes de la ecografía prenatal, como uropatías obstructivas, cardiopatías congénitas y otras malformacio- nes internas, que antes de la ecografía se descubrían más tardíamente. Sin embargo, hay otras como el síndrome de Down, que por lo general no se diagnostica antenatalmente, aún cuando hay signos ultrasonográficos que pueden hacerlo sospechar, que pareciera que están aumentando su frecuencia. En Chile, de acuerdo a los datos publicados, la prevalencia al nacimiento del síndrome de Down está, en la actualidad, por sobre 2,2 por 1000 nacimientos ${ }^{7}$. Esta tendencia la hemos comunicado en trabajos anteriores ${ }^{8,9}$ y hemos confirmado la correlación que existe con el aumento del promedio de edad materna en nuestra maternidad ${ }^{9-12}$. El ECLAMC da para el período 1982-2001 una tasa de 1,63 por mil (5 841/3 $574609^{10}$ ) y para el período 1995-2008 una prevalencia de 1,9 por mil nacimientos.

Se ha relacionado al síndrome de Down con factores de riesgo tan variados como agentes genéticos, físicos, químicos, inmunológicos, infecciosos y sociales, pero solamente tres se consideran en la actualidad como probables agentes etiológicos de la trisomía 21: ellos son anomalías cromosómicas de los padres capaces de inducir una no-disyunción meiótica secundaria, la exposición precigótica materna a radiaciones ionizantes y la edad materna avanzada. Las dos primeras, si bien son importantes, son responsables, en la práctica, de una minoría de casos de síndrome de Down, 
mientras que se ha demostrado que existe una estrecha relación entre esta afección y la edad materna $^{9-12}$. Un tercio de los niños afectados con trisomía 21 nacen de madres de 40 años o más.

Por la importancia médica y sobre todo social, es que siempre ha sido una preocupación permanente de los diferentes programas de registro y monitoreo dedicados a estudiar este problema. Esto nos ha motivado a estar siempre alertas a cualquier cambio que se pueda producir en las prevalencias al nacimiento de esta trisomía y publicando nuestros hallazgos con el fin de crear conciencia tanto en los médicos, funcionarios de la Salud, como en el público en general de los factores de riesgos que influyen en la aparición de nuevos casos. En repetidas ocasiones hemos comunicado nuestra experiencia y la de otros autores al respecto $^{1-6}$ con el fin de educar y advertir de estos peligros. El ECLAMC publicó un Decálogo para la prevención primaria de las malformaciones congénitas y en el $\mathrm{N}^{\circ} 2$ propone que la familia debe completarse mientras se es joven ${ }^{14}$.

La frecuencia del síndrome de Down en los diferentes países o en diferentes regiones de un mismo país, puede verse afectada por la diferente distribución de las edades maternas, pero también por el diagnóstico prenatal seguido de aborto electivo, en los países en que está legalmente permitido. De ahí que haya diferencias importantes al compararlos. Sin embargo, esta tendencia al aumento se ha visto en programas de países como Finlandia, Australia, Estrasburgo, Canadá en los que se registra tanto los nacidos vivos como los productos de estos abortos selectivos ${ }^{14}$.

\section{Objetivos}

Comprobar la tendencia en Chile y Latino América del aumento de las tasas de prevalencia al nacimiento del síndrome de Down, así como la disminución de ellas en el resto del mundo.

\section{Pacientes y Método}

Se estudiaron tres muestras: la primera la constituye el material registrado en la mater- nidad del Hospital Clínico de la Universidad de Chile (HCUCH) desde 1972 a 2009; la segunda formada por los nacimientos en los hospitales chilenos activos participantes del ECLAMC y la tercera todos los casos nacidos en los 180 hospitales de 9 países participantes del ECLAMC.

Se comparó con las frecuencias de otros Programas del resto del mundo participantes del International Clearinghouse for Birth Defects Monitoring Systems ${ }^{15,16}$.

Las comparaciones entre las tasas se hicieron con prueba de $\chi^{2}$. Se hizo un análisis de regresión y correlación lineal de la tasa de prevalencia al nacimiento de síndrome de Down.

\section{Resultados}

En el período 1972-2009 ocurrieron 145207 nacimientos en la maternidad del HCUCH y se diagnosticaron 286 casos de síndrome de Down, lo que representa una tasa global de 1,96 por mil.

La tabla 1 presenta la evolución de las Tasas de prevalencia al nacimiento del síndrome de Down en la Maternidad del Hospital Clínico de la Universidad de Chile en el período 19722009. Podemos observar como estas tasas han aumentado significativamente desde 1,03 por mil nacimientos en 1972 a 2,93 en el 2009 ( $p=0,056$ al comparar 1972 con 2009). Se construyó la recta de regresión mínimo cuadrática de las tasas de síndrome de Down a través de los años, obteniéndose una pendiente positiva de $0,069(\mathrm{p}=0,02)$ y un coeficiente de correlación de Pearson de 0,48 ( $\mathrm{p}=0,001)$.

Esto es lo que hemos observado en el HCUCH. Presentaremos a continuación la experiencia de lo que ha ocurrido en los otros hospitales chilenos participantes en el ECLAMC.

Las tasas de prevalencia de los otros hospitales chilenos son muy homogéneas como se puede apreciar en la tabla 2. Todas son más altas que el promedio del ECLAMC, especialmente las de los hospitales de Rancagua, Curicó y Linares que son sinificativamente mayores ( $p<0,05)$. Lo mismo ocurre con el Hospital San Borja Arriarán, Naval de Valpa- 
NAZER J. y cols.

Tabla 1. Evolución de las tasas anuales y promedio de edad materna de niños con síndrome de Down y del total de las madres de la maternidad del Hospital Clínico Universidad de Chile, Período1972 a 2009

\begin{tabular}{|c|c|c|c|c|c|}
\hline Año & $\begin{array}{c}\text { Total } \\
\text { nacimientos }\end{array}$ & RN afectados & Tasa $\mathbf{x}$ mil & $\begin{array}{c}\text { Edad materna } \\
\text { Down }\end{array}$ & $\begin{array}{l}\text { Edad materna } \\
\text { total madres }\end{array}$ \\
\hline 1972 & 12530 & 13 & 1,03 & & 25,00 \\
\hline 1973 & 11153 & 15 & 1,34 & & 24,83 \\
\hline 1974 & 11043 & 16 & 1,44 & & 25,05 \\
\hline 1975 & 7393 & 13 & 1,75 & & 25,20 \\
\hline 1976 & 6998 & 9 & 1,28 & & 25,05 \\
\hline 1977 & 5780 & 11 & 1,90 & 32,5 & 24,39 \\
\hline 1978 & 2920 & 8 & 2,74 & 30.5 & 25,37 \\
\hline 1979 & 3662 & 5 & 1,36 & 28,4 & 24,97 \\
\hline 1980 & 4043 & 10 & 2,47 & 29,8 & 24,99 \\
\hline 1981 & 4176 & 9 & 2,15 & 31,3 & 25,24 \\
\hline 1982 & 3804 & 7 & 1,84 & 33,0 & 25,51 \\
\hline 1983 & 4519 & 8 & 1,77 & 29,1 & 25,93 \\
\hline 1984 & 3889 & 5 & 1,29 & 28,0 & 26,16 \\
\hline 1985 & 4321 & 7 & 1,62 & 29,9 & 26,10 \\
\hline 1986 & 3665 & 2 & 0,65 & 34,1 & 26,20 \\
\hline 1987 & 3739 & 6 & 1,60 & 29,7 & 27,08 \\
\hline 1988 & 2961 & 7 & 2,36 & 32,2 & 27,38 \\
\hline 1989 & 2820 & 7 & 2,48 & 33,9 & 27,16 \\
\hline 1990 & 3748 & 8 & 2,1 & 28,8 & 26,77 \\
\hline 1991 & 4160 & 9 & 2,16 & 34,0 & 26,50 \\
\hline 1992 & 3416 & 10 & 2,93 & 39,9 & 26,52 \\
\hline 1993 & 2281 & 8 & 3,07 & 30,5 & 27,05 \\
\hline 1994 & 256 & 5 & 1,96 & 36,5 & 27,29 \\
\hline 1995 & 2650 & 3 & 1,13 & 34,3 & 27,80 \\
\hline 1996 & 2355 & 5 & 2,12 & 32,0 & 28,20 \\
\hline 1997 & 2485 & 7 & 2,82 & 36,1 & 28,31 \\
\hline 1998 & 2917 & 8 & 2,74 & 38,0 & 28,77 \\
\hline 1999 & 2267 & 4 & 1,76 & 33,0 & 28,25 \\
\hline 2000 & 2208 & 6 & 2,71 & 32,8 & 29,59 \\
\hline 2001 & 2160 & 6 & 2,77 & 33,0 & 29,37 \\
\hline 2002 & 1840 & 9 & 4,89 & 33,0 & 29,77 \\
\hline 2003 & 1584 & 14 & 8,83 & 37,0 & 26,84 \\
\hline 2004 & 1539 & 7 & 4,55 & 37,9 & 29,26 \\
\hline 2005 & 1522 & 4 & 2,62 & 34,5 & 27,77 \\
\hline 2006 & 1492 & 2 & 1,34 & 36,5 & 29,57 \\
\hline 2007 & 1586 & 5 & 3,15 & 34,2 & 29,30 \\
\hline 2008 & 1654 & 4 & 2,42 & 33,8 & 28,60 \\
\hline 2009 & 1367 & 4 & 2,93 & 39,7 & 29,39 \\
\hline
\end{tabular}




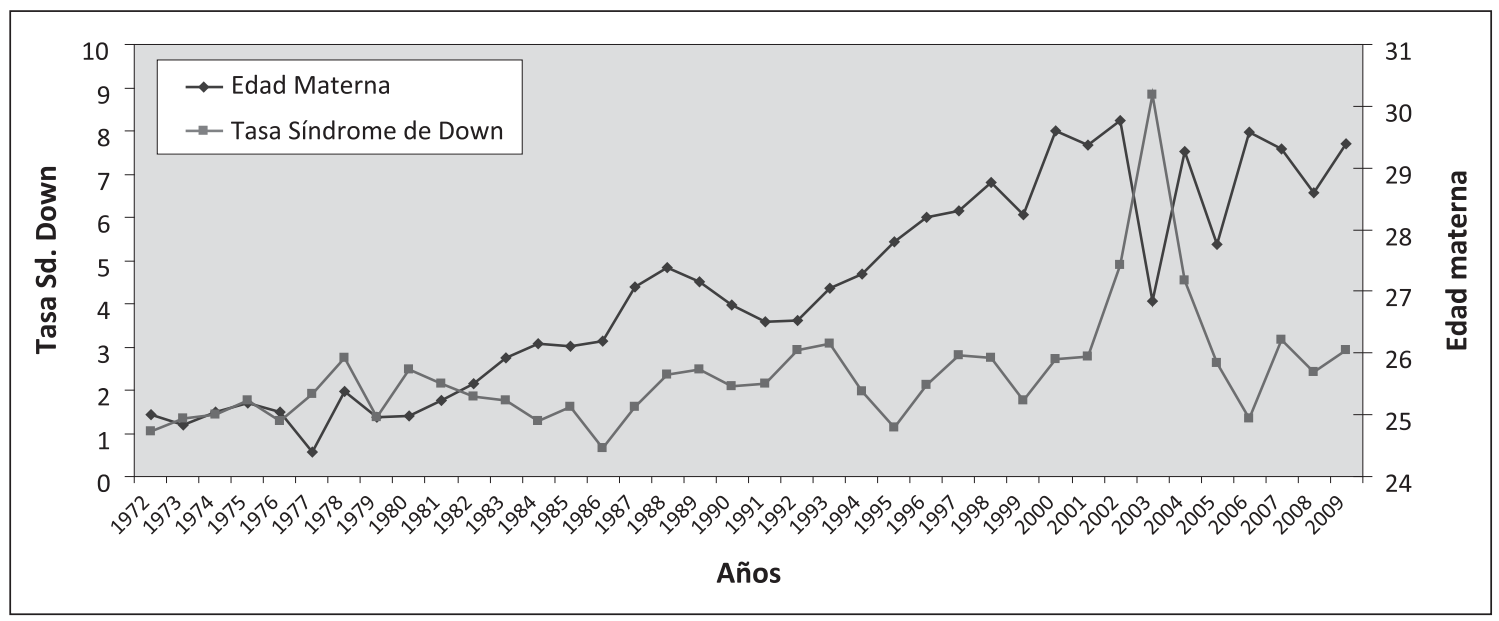

Figura 1. Evolución edad materna y tasa por 1000 de síndrome de Down HCUCH.

Tabla 2. Tasas de prevalencia al nacimiento de síndrome de Down en los hospitales chilenos participantes del ECLAMC. Período 1998-2005

\begin{tabular}{|lc|}
\hline Hospital & $\begin{array}{c}\text { Tasa por } \mathbf{1 0 0 0} \\
\text { nacimientos }\end{array}$ \\
\hline Curicó & 2,86 \\
\hline Rancagua & 2,72 \\
\hline Linares & 2,68 \\
\hline San Borja Arriarán & 2,60 \\
\hline Naval de Valparaíso & 2,49 \\
\hline Sótero del Río & 2,48 \\
\hline Sanatorio Alemán de Concepción & 2,34 \\
\hline San José & 2,23 \\
\hline Valdivia & 1,90 \\
\hline Clínico Universidad de Chile & 1,88 \\
\hline Promedio de Chile & 2,41 \\
\hline Promedio del ECLAMC & 1,88 \\
\hline
\end{tabular}

Tabla 3. Evolución de las tasas de prevalencia al nacimiento de malformaciones congénitas en Latino América según un informe del International Clearinghouse for Birth Defects Monitoryng Systems ${ }^{14,15}$

\begin{tabular}{|cc|}
\hline Período & Tasa por $\mathbf{1} \mathbf{0 0 0}$ \\
\hline $1974-1979$ & 1,46 \\
$1985-1989$ & 1,47 \\
$1990-1994$ & 1,58 \\
$1995-1996$ & 1,82 \\
1998 & 1,88 \\
\hline
\end{tabular}

raíso, Clínica Francesa de Concepción y Hospital San José de Santiago. El promedio para todos los hospitales chilenos participantes según informe del ECLAMC fue de 1,96 por mil nacimientos para el período 1982-2001 y 2,47 para el período 1995-2008.

El único registro a nivel nacional que comprende a todos los nacimientos de una Región es el Registro del Maule, reconocido como miembro del ICBDSR; en el período 20022009 mantuvo sus frecuencias, con algunas variaciones, estuvieron entre 1,5 y 1,9 por mil nacimientos (rango 1,3 - 3,1).

En Latinoamérica, según datos del ECLAMC, la tendencia en todos los países participantes es aumentar las tasas de SD. Según el Internacional Clearinghouse for Birth Defects Monitoring Systems ${ }^{15,16}$, en el período 1974-79 en Sudamérica la tasa por 1000 nacimientos era de 1,46 . Subió a 1,49 por mil entre 198589, a 1,58 entre 1990-94, a 1,82 en el período 1995-96 para alcanzar 1,85 en 1998 (tabla 3). El ECLAMC da para el período 1998-2005 una Tasa Global de 1,88\%. Sobre ese promedio están las tasas de Chile (2,47 por 10 000), Argentina $(2,01)$ y Paraguay $(1,98)$. Bajo él están Brasil (1,72), Colombia (1,72), Bolivia $(1,55)$, Venezuela $(1,49)$, Ecuador $(1,48)$ y Uruguay $(1,32)$ (tabla 4). 
Tabla 4. Tasas de prevalencia al nacimiento de síndrome de Down de los países Sudamericanos del ECLAMC. Período 1998-2005

\begin{tabular}{|lc|}
\hline País & $\begin{array}{c}\text { Tasa por mil } \\
\text { nacimientos }\end{array}$ \\
\hline Chile & 2,47 \\
\hline Argentina & 2,01 \\
\hline Paraguay & 1,98 \\
\hline Brasil & 1,72 \\
\hline Colombia & 1,72 \\
\hline Bolivia & 1,55 \\
\hline Venezuela & 1,49 \\
\hline Ecuador & 1,48 \\
\hline Uruguay & 1,32 \\
\hline Hospital Clínico Universidad de Chile & 1,88 \\
\hline Total del ECLAMC & 1,88 \\
\hline
\end{tabular}

\section{Discusión}

En el Hospital Clínico de la Universidad de Chile ha habido un incremento significativo de la tasa de síndrome de Down en el período 1972-2009 ( $<$ < 0,056). Sin embargo, llama la atención lo ocurrido entre el año 2002 y 2004, donde se produjo un alza importante de la tasa desde 2,77 en 2001 a 4,89 en el 2002, 8,83 en el 2003 para bajar a 4,55 en el 2004 y volver 2,62 en el 2005. No tenemos una explicación para esto. Ha habido una disminución apreciable del número de partos en la Maternidad del Hospital Clínico al mismo tiempo que un aumento en el número de casos de SD sin que haya un amento de los diagnósticos prenatales.

Existe una gran heterogeneidad de frecuencias entre los países sudamericanos. Seguramente hay factores como edad materna, etnias que influyen en ello. Al analizar la edad materna como factor de riesgo para esta patología, pareciera que siendo de gran importancia como ha sido demostrado en múltiples comunicaciones ${ }^{7,9-12}$, no sería la única causa de estas diferencias. En el período 1998-2005 la mayor proporción de mujeres de 35 años o más que tuvieron hijos se encontró en Chile con 14,4\% seguido de Uruguay con $13,6 \%$ y ocurre que Chile presentó, como era de esperar, la tasa más alta de $\mathrm{S}$ de Down, 24,7 por 10 000, mien- tras que Uruguay la más baja, 13,2 por 10000 nacimientos (tabla 5).

En lo que se refiere a la evolución de las tasas de prevalencia al nacimiento de SD en el resto del mundo, es interesante verlo antes y después de que en algunos países se permitió legalmente eliminar los fetos portadores de este síndrome. Por lo tanto, es distinto lo que se encuentre en Latinoamérica donde no existe la posibilidad legal de hacerlo y otros países, especialmente europeos y asiáticos donde sí se produjo esta situación.

En efecto la Dra. María Luisa MartínezFrías Directora del ECEMC (Estudio Colaborativo Español de Malformaciones Congénitas) que tiene un programa similar al del ECLAMC dice que la frecuencia de síndrome de Down en el ECEMC es de 0,77 recién nacidos por cada 1000 nacimientos en madres menores de 35 años. Por el contrario a partir de los 35 años se inicia un incremento progresivo que llega a ser 10 veces superior (8,0 por mil nacimientos). Agrega que en España durante los años 1980-1985 (antes de la posibilidad legal de interrumpir la gestación, la frecuencia global de recién nacidos con $\mathrm{S}$ de Down era de 1,48 por 1000 . En la actualidad ha pasado a ser de 0,64 por mil, lo que corresponde a una reducción de $45 \%$. Esta cifra está a menos de una décima para ser considerada dentro de las Enfermedades Raras (0,5 por 1000 o menores) $)^{15}$.

Los nacimientos de niños con síndrome de Down han experimentado una caída brusca en el último cuarto de siglo en España. La mejoría de las técnicas de diagnóstico prenatal y la aprobación de la actual Ley del Aborto han propiciado que en 2006 se registrase un 56\% menos de casos que en 1980. Este descenso ha sido aún más drástico en el caso de los recién nacidos cuyas madres son mayores de 35 años: 85\% menos. Estos datos proceden del Estudio Colaborativo Español de Malformaciones Congénitas (ECEMC), que no cubre todos los nacimientos, pero constituye el registro más amplio en ese país ${ }^{15}$.

La razón de la mayor reducción entre las madres añosas radica en que, por ser el grupo etario con más riesgo, se les ofrece de forma sistemática la realización de una amniocente- 
SÍNDROME DE DOWN

Tabla 5. Distribución de los nacimientos según grupos etarios de sus madres, por país y Total del ECLAMC. Período 1998-2005

\begin{tabular}{|c|c|c|c|c|c|c|c|}
\hline $\begin{array}{l}\text { Grupos de } \\
\text { edades }\end{array}$ & $<=19$ años & $\%$ & 20 a 34 años & $\%$ & $>=35$ años & $\%$ & Total \\
\hline Argentina & 156856 & 19,2 & 568721 & 69,7 & 89877 & 11,0 & 815454 \\
\hline Bolivia & 19387 & 18,1 & 75011 & 69,1 & 12886 & 12,0 & 107284 \\
\hline Brasil & 104946 & 20,3 & 356295 & 68,0 & 56601 & 10,9 & 517842 \\
\hline Colombia & 15902 & 23,3 & 44987 & 66,0 & 7261 & 6,3 & 68150 \\
\hline Chile & 65565 & 17,5 & 255233 & 68,1 & 53842 & 14,4 & 374640 \\
\hline Ecuador & 27302 & 20,0 & 94897 & 69,7 & 13948 & 10,2 & 136147 \\
\hline Paraguay & 5820 & 21,7 & 17779 & 63,3 & 3206 & 12,0 & 26805 \\
\hline Uruguay & 14688 & 15,6 & 67034 & 71,0 & 12777 & 13,6 & 94449 \\
\hline Venezuela & 59652 & 25,3 & 15803 & 67,0 & 18509 & 7,8 & 236193 \\
\hline Total ECLAMC & 475711 & 19,7 & 1660620 & 68,9 & 273076 & 11,3 & 2409407 \\
\hline
\end{tabular}

$\mathrm{p}<0,001$.

sis, con estudio citogenético, que es la prueba diagnóstica más certera. La mayoría de ellas acepta este procedimiento invasivo que presenta un riesgo de aborto espontáneo de entre el $0,5 \%$ y el $1 \%$ y, cuando se detecta la anomalía, el 95\% de esas mujeres opta por la interrupción voluntaria del embarazo ${ }^{15}$.

Según el Informe anual del Internacional Clearinghouse for Birth Defects Monitoring System (ICBDSR) ${ }^{15}$ que muestra la evolución de las tasas de prevalencia al nacimiento del síndrome de Down en los 20 Programas participantes en el entre 1974 y 1998, se puede apreciar que mientras algunos Programas presentan un aumento importante de sus tasas, como Francia, Strasburgo, Irlanda, Canadá-Alberta, Australia, Japón, Nueva Zelanda, Sudamérica, otros, por el contrario, han disminuido considerablemente sus tasas, como Hungría, Israel, Italia, Holanda y España.

Sin embargo, en un nuevo informe del ICBDSR, ahora del período $2001-2005^{16}$ comunica que sólo en los Registros de Maule en Chile, el total del ECLAMC y Francia REMERA, hubo un aumento significativo de las frecuencias de SD. Los otros Programas que aumentaron: Malta, República Checa, Suecia, Australia W, Finlandia, Canadá Alberta y USA Utah no alcanzaron significancia. Todos los demás bajaron significativamente sus tasas. Las bajas más llamativas son España a 0,60 por 1000 nacimientos e Irán a 0,30 por 1000.

En lo que se refiere a la evolución de las tasas de prevalencia al nacimiento de SD en el resto del mundo, es interesante verlo antes y después de que en algunos países se permitió legalmente eliminar los fetos portadores de este síndrome. Por lo tanto, es distinto lo que se encuentre en Latinoamérica donde no existe la posibilidad legal de hacerlo y otros países, especialmente europeos y asiáticos donde sí se produjo esta situación.

Gocchi, con material de los programas del Internacional Cleaaringhouse ${ }^{16}$, comunica que ha aumentado la proporción de madres de 35 años y más al mismo tiempo que aumenta la tasa global de síndrome de Down (Nacidos vivos, mortinatos y producto de abortos electivos) de 13,1 a 18,2 por 10000 nacimientos en el período 1993-2004. Sin embargo, la tasa sólo en nacidos vivos se ha mantenido estable (8,3 por 10000$)$ debido al gran incremento de los abortos electivos en esos países. En Programas de Francia, Italia, República Checa se ha observado una disminución de las tasas de SD al mismo tiempo que se observa un gran incremento de los abortos electivos. En algunos Programas como Alberta en Canadá hubo un incremento en ese período. 


\section{Conclusión}

La evolución de las Tasas de prevalencia al nacimiento de síndrome de Down es muy diferente tanto el plano local y regional, entre países de la misma región y de regiones distintas. En Chile, las Tasas son superiores al promedio del ECLAMC. En Sudamérica hay una tendencia al aumento, promedio 2,89 por mil en el período 2001-2005 según datos del ECLAMC y en Europa y Asia a disminuir significativamente, donde las tasas han descendido a cifras extremas como Irán que tiene 0,3 por mil nacimientos y España 0,6 por mil. Esto debido, seguramente, a la legalización del aborto electivo, lo que no está legalmente permitido en Chile y resto de Latino América.

\section{Referencias}

1.- Nazer J, Díaz G, Pizarro MT: Malformaciones congénitas: Estudio Clínico y Epidemiológico. Pediatría Santiago 1978; 26: 295-302.

2.- $\quad$ Nazer J, Cifuentes L, Ruiz G: Incidencia de 50 malformaciones congénitas en 8 maternidades chilenas participantes en el Estudio Colaborativo Latino Americano de Malformaciones Congénitas (ECLAMC). Período 1982-1988. Rev Chil Pediatr 1991; 62: 37-44.

3.- Nazer J, Cifuentes L, Ruiz G: ¿Están aumentando las malformaciones congénitas? Estudio comparativo entre los períodos 1971-1977 y 182-1991. Rev Med Chile 1993; 121: 1968-74.

4.- Nazer J, Cifuentes L, Meza M: Incidencia de malformaciones congénitas en 10 maternidades chilenas participantes del ECLAMC. Comparación e 3 períodos: 1971-1977, 1982-1988 y 1989-1999. Rev Med Chile 1997; 125: 993-1001.

5.- Nazer J, Aravena T, Cifuentes L: Malformaciones Congénitas un problema emergente. Rev Med Chile 2001; 129: 895-904.

6.- $\quad$ Nazer J, Ramírez C, Cifuentes L: 38 años de vigilancia epidemiológica de labio Leporino y paladar hendido en la maternidad del hospital Clínico de l Univeridad de Chile. Período 1971-2008. Rev Med Chile 2010;

7.- Nazer J, Antolini M, Juárez ME, Cifuentes L, Hübner $M E$, Pardo RA: Prevalencia al nacimiento de aberraciones cromosómicas en el Hospital Clínico de la Universidad de Chile. Período 1990-2000. Rev Med Chile 2003; 131: 651-58.

8.- Nazer J, Castillo S, Cifuentes L, Ruiz G, Pizarro MT, Parada $L$ : Fecuencia de las malfomaciones congenitas en Chile. Peíodo 1969-1986. Resultado de un Estudio Colaborativo Latino Americano. Rev Med Chile 1989; 117: 219-27.

9.- Nazer J, Hübner ME, Cifuentes L, Ramírez R, Catalán $J$, Ruiz G: Aumento de la incidencia del síndrome de Down y su posible relación con el incremento de la edad materna. Rev Med Chile 1991; 119: 465-71.

10.- Nazer J, Cifuentes L, Ruiz G, Pizarro MT: Edad materna como factor de riesgo para malformaciones congénitas. Rev Med Chile 1994; 122: 299-303.

11.- Nazer J, Eaglin MA, Ciuentes L: Incidencia del síndrome de Down en la maternidad del Hospital Clínico de la Universidad de Chile. Un registro de 25 años. Rev Med Chile 1998; 126: 382-90.

12.- Nazer J, Aguila A, Cifuentes L: Vigilancia epidemiológica del síndrome de Down en Chile, 1972 a 2005. Rev Med Chile 2006; 134: 1549-57.

13.- Nazer J: Anomalías congénitas estructurales en el Recién Nacido (Segunda parte): Prevención de las malformaciones congénitas. Rev Hospital Clínico Universidad de Chile 2002; 13: 294-305.

14.- Down Síndrome. En Congenital Malformations Worldwide. A report from The International Clearinghouse for Birth Defects Monitoring Systems. Ed Elsewier. 1991; 1157-63.

15.- Martínez-Frías ML: Situación del síndrome de Down en nuestro país. Aclaración conceptual sobre su frecuencia y visibilidad. http://www.madrimasd.org. 2010.

16.- Cocchi G, Gualdi S, Bower C, et al: International Trends of Down Sindrome 1993-2004 Birth relation to maternal age an Terminations of pregnancies. Birth Defects Research (Part A). 2010. 88: 474-9. 